Kazuki Komatsu • Nobukatsu Nakamura

Mohsen Ghadami • Naomichi Matsumoto

Tatsuya Kishino • Tohru Ohta • Norio Niikawa

Koh-ichiro Yoshiura

\title{
Confirmation of genetic homogeneity of nonsyndromic low-frequency sensorineural hearing loss by linkage analysis and a DFNA6/14 mutation in a Japanese family
}

Received: March 28, 2002 / Accepted: April 18, 2002

\begin{abstract}
Nonsyndromic low-frequency sensorineural hearing loss (LFSNHL) comprises a group (DFNA1, DFNA6, DFNA14, and DFNA38) of hearing disorders affecting only frequencies below $2000 \mathrm{~Hz}$, and is often associated with tinnitus. An LFSNHL locus has recently been assigned to chromosome 4p16, and mutations in WFS1, the causative gene for Wolfram syndrome, have been found to cause LFSNHL in families with DFNA6, DFNA14, or DFNA38. We performed a genome-wide linkage analysis of a Japanese family in which 20 members were affected with LFSNHL and obtained a maximum LOD score of 5.36 at a recombination fraction of $0.05(P=1.00)$ at the $D 4 S 2983$ locus on $4 \mathrm{p} 16$. Haplotype analysis revealed that the disease locus mapped to between D4S2366 and D4S2983. Mutation analysis revealed a novel missense mutation (K634T) in WFS1. We thus concluded that the LFSNHL in this family was caused by the WFS1 mutation. The mutation observed (K634T) was located in the hydrophobic, extracytoplasmic, juxta-transmembrane region of the WFS1 protein, wolframin, and was hitherto undescribed. This unique mutation site in our patients is likely related to their milder phenotype (lacking tinnitus) compared with those of six previous DFNA6/14 patients with WFS1 mutations. It is likely that a
\end{abstract}

K. Komatsu - M. Ghadami - N. Matsumoto · N. Niikawa

K.-i. Yoshiura $(\square)$

Department of Human Genetics, Nagasaki University School of

Medicine, 1-12-4 Sakamoto, Nagasaki 852-8523, Japan

Tel. +81-95-849-7120; Fax +81-95-849-7121

e-mail:kyoshi@net.nagasaki-u.ac.jp

K. Komatsu

Department of Otolaryngology, Nagasaki University School of Medicine, Nagasaki, Japan

K. Komatsu $\cdot$ M. Ghadami $\cdot$ N. Matsumoto $\cdot$ T. Kishino $\cdot$

N. Niikawa $\cdot$ K.-i. Yoshiura

CREST, Japan Science and Technology Corporation, Kawaguchi, Japan

N. Nakamura

Shin-Uonome Clinic, Nagasaki, Japan

T. Kishino $\cdot$ T. Ohta

Gene Research Center, Nagasaki University, Nagasaki, Japan genotype-phenotype correlation is also applicable in the case of DFNA6/14/38.

Key words Low-frequency sensorineural hearing loss . Linkage analysis - DFNA6/14/38 - Novel mutation - WFS1

\section{Introduction}

Nonsyndromic sensorineural hearing loss affecting high frequencies comprises a group of relatively common, genetically heterogeneous disorders. In contrast, low-frequency $(<2000 \mathrm{~Hz})$ sensorineural hearing loss (LFSNHL) comprises an unusual group of hearing disorders. Monogenic and prelingual nonsyndromic sensorineual hearing impairment occurs in approximately 1 in 2000 births. The autosomal dominant inheritance pattern has been reported as $20 \%-25 \%$ (Cohen and Gorlin 1995). However, the prevalence of LFSNHL is unknown. Many patients with LFSNHL have mild tinnitus without any associated features such as vertigo. Because high-frequency hearing is generally preserved in patients with LFSNHL, such patients usually have only a little difficulty understanding speech, unless presbycusis or noise exposure has caused high-frequency hearing loss in later life. Consequently, LFSNHL is often asymptomatic, and many patients do not wear hearing aids. Historically, four autosomal dominant types of hearing loss (DFNA1, DFNA6, DFNA14, and DFNA38) have been reported to be associated with LFSNHL among more than 70 mapped loci of nonsyndromic sensorineural hearing loss. DFNA1 is clinically and genetically different from the others, because it is caused by a mutation of the Homo sapiens diaphanous homolog 1 gene (DIAPH1) located at 5q31 (Lynch et al. 1997), whereas DFNA6, DFNA14, and DFNA38 are reported to be caused by mutations of the gene WFS1 at 4p16.3 (Bespalova et al. 2001; Young et al. 2001), the causative gene for Wolfram syndrome (Strom et al. 1998).

We recently encountered a Japanese family in which 20 members were affected with LFSNHL. Here, we report the 
results of linkage and mutation analyses on genomic DNA from this family.

\section{Subjects and methods}

Family and patients

The family with LFSNHL studied comprised 42 individuals, including 20 affected members in four generations (Fig. 1a). Following informed consent, a total of 36 family members (15 affected and 21 unaffected persons) joined a clinical evaluation study, which included audiometric analysis (Fig. 1b). Genomic DNA was extracted from 30 family members by the standard method and used for linkage and mutation analyses (Sambrook and Russel 2001).

Linkage and haplotype analyses

Samples from thirty family members were subjected to a genome-wide linkage analysis by using an ABI Prism Linkage Mapping Set-MD10 (PE Applied Biosystems, Foster City, CA, USA). In addition, highly polymorphic microsatellite markers located around D4S431 were retrieved from the U.S. National Center for Biotechnology Information (NCBI) Genome Database (http://www. ncbi.nlm.nih.gov) and used for fine mapping. Polymerase chain reaction (PCR) products were run on an ABI automated sequencer model 377 (PE Applied Biosystems), and allele types were scored with GenoTyper software (version 2.0, Applied Biosystems). A two-point linkage analysis was performed with the computer program MLINK (FASTLINK software, version 4.1P) (Cottingham et al. 1993; Schaffer et al. 1994). Deafness in the family was modeled as autosomal dominant with complete penetrance $(P=$ $1.00)$ and disease-allele frequency of $<0.001$. We used the observed number of alleles $(\mathrm{N})$ in the pedigree for LOD score calculations and set allele frequencies as $1 / \mathrm{N}$.

Mutation analysis

Nucleotide sequences of ten genes (FLJ11230, HMGE, KIAA0232, KIAA0935, KIAA1322, LOC93623,
LOC114923, PGR1, and S100P) and their exon/intron boundaries located in the mapped disease region of the family were obtained from GenBank. Similarly, the sequences of WFS1 and its exon/intron boundaries were also obtained from GenBank (accession numbers, XM_003395, AC004599, and AC004689). Oligonucleotide primers were designed to cover the coding sequences of the ten genes as well as that of WFS1 (Table 1). PCR was performed in a 20$\mu \mathrm{l}$ reaction mixture containing $20 \mathrm{ng}$ genomic DNA, $1 \mu \mathrm{M}$ of each primer, $200 \mu \mathrm{M}$ of each dNTP, $10 \mathrm{mM}$ Tris- $\mathrm{HCl}(\mathrm{pH}$ $8.3), 50 \mathrm{mM} \mathrm{KCl}, 1.5 \mathrm{mM} \mathrm{MgCl}_{2}, 0.001 \%$ gelatin, and $0.5 \mathrm{U}$ AmpliTaq Gold (PE Applied Biosystems) by using a GeneAmp PCR System 9700 (PE Applied Biosystems). PCR conditions were as follows: one cycle at $94^{\circ} \mathrm{C}$ for $10 \mathrm{~min}, 40$ cycles at $94^{\circ} \mathrm{C}$ for $30 \mathrm{~s}$ for denaturation, $55^{\circ} \mathrm{C}$ for $30 \mathrm{~s}$ for annealing, and $72^{\circ} \mathrm{C}$ for $30 \mathrm{~s}$ for extension, and another step at $72^{\circ} \mathrm{C}$ for $10 \mathrm{~min}$ to ensure complete extension. PCR products were purified with a QIAquick PCR purification kit (QIAGEN, Valencia, CA, USA) and sequenced directly with a Big-Dye Terminator Cycle Sequencing Kit on an ABI3100 automated sequencer (PE Applied Biosystems). Sequences in the patients were compared with those of normal control persons.

\section{Results}

Clinical and audiometric findings

Clinical manifestations including audiometric patterns (Fig. 1b) of the affected individuals in the family revealed that their LFSNHL was consistent with DFNA6/14-type hearing impairment, although they never had any tinnitus. As no affected individuals had juvenile-onset diabetes mellitus or optic atrophy, they did not suffer from Wolfram syndrome.

Genetic findings

Segregation analysis revealed that the LFSNHL of the family was consistent with autosomal dominant inheritance with complete penetrance and a segregation ratio of $0.563 \pm$ 0.008 , under the condition of complete selection of patients.

Table 1. Sequences for primers used for the present WFS1 mutation search

\begin{tabular}{lll}
\hline Name & Sense strand $\left(5^{\prime}-3^{\prime}\right)$ & Antisensense strand $\left(5^{\prime}-3^{\prime}\right)$ \\
\hline ORF2 & AAGCGGTGCTGGCCCATG & CCGTTCCCACCCAGCTATC \\
ORF3 & TACTCCTGGCCTGGATTTGA & CATGGGCACCCTACCAACA \\
ORF4 & AGTGGCCGGAGGTCAGT & CCAACAGCATCACCAGCGT \\
ORF5 & AGTCAGATGTCCATGTCCATGCATCC & CTCTACAGGAAGGTTCTGGT \\
ORF6 & GAGCACGCTACGTGGTGCT & GGAGGCACGGGTGAGATAG \\
ORF7 & TCCGAGACGACCTGAG & GCGCGTTGATGTGGTGCG \\
ORF8a & TCACGCTGGTAGAAGGTGG & GTCGGTGAGGGTGCGGAA \\
ORF8b & GCTGGTCATGTTCTACCTGT & TGCCATGCGGAAGAAGAGTA \\
ORF8c & GACCTTCATCACCGTGCCT & GAACCAGCAGAACAGCACG \\
ORF8d & AGGCCAGCTTCTCTGTGGT & GCTTGGCCAGCAGCTTAAG \\
ORF8e & AGGCCTACCCTGCCTGCA & TTCACGGCGCCATGCACG \\
ORF8f & GCATGGCCCAGCTCTCAC & GTGGAGATGGCATGCAATC \\
\hline ORF,
\end{tabular}

ORF, open reading frame 
Fig. 1. a Pedigree of the family depicting haplotypes at marker loci on chromosome 4. Solid squares/circles show males/females, respectively, affected with low-frequency sensorineural hearing loss (LFSNHL). Open squares/circles show unaffected males/females, respectively. Numbers in open boxes indicate a putative disease haplotype, and short horizontal lines depict recombination sites. Haplotypes in parentheses $(I-1, I-2, I I-1, I I-3$, and $I I I-5)$ were deduced from those of their offspring. Symbols with slashes through them indicate deceased individuals. Asterisks show possible mutated alleles. b Pure tone audiogram of a 17-year-old girl (III-20) with LFSNHL (DFNA6/ 14). Masked bone conduction curves nearly follow air conduction curves. Crosses and circles indicate air conduction in the left and right ears, respectively
I

a

a
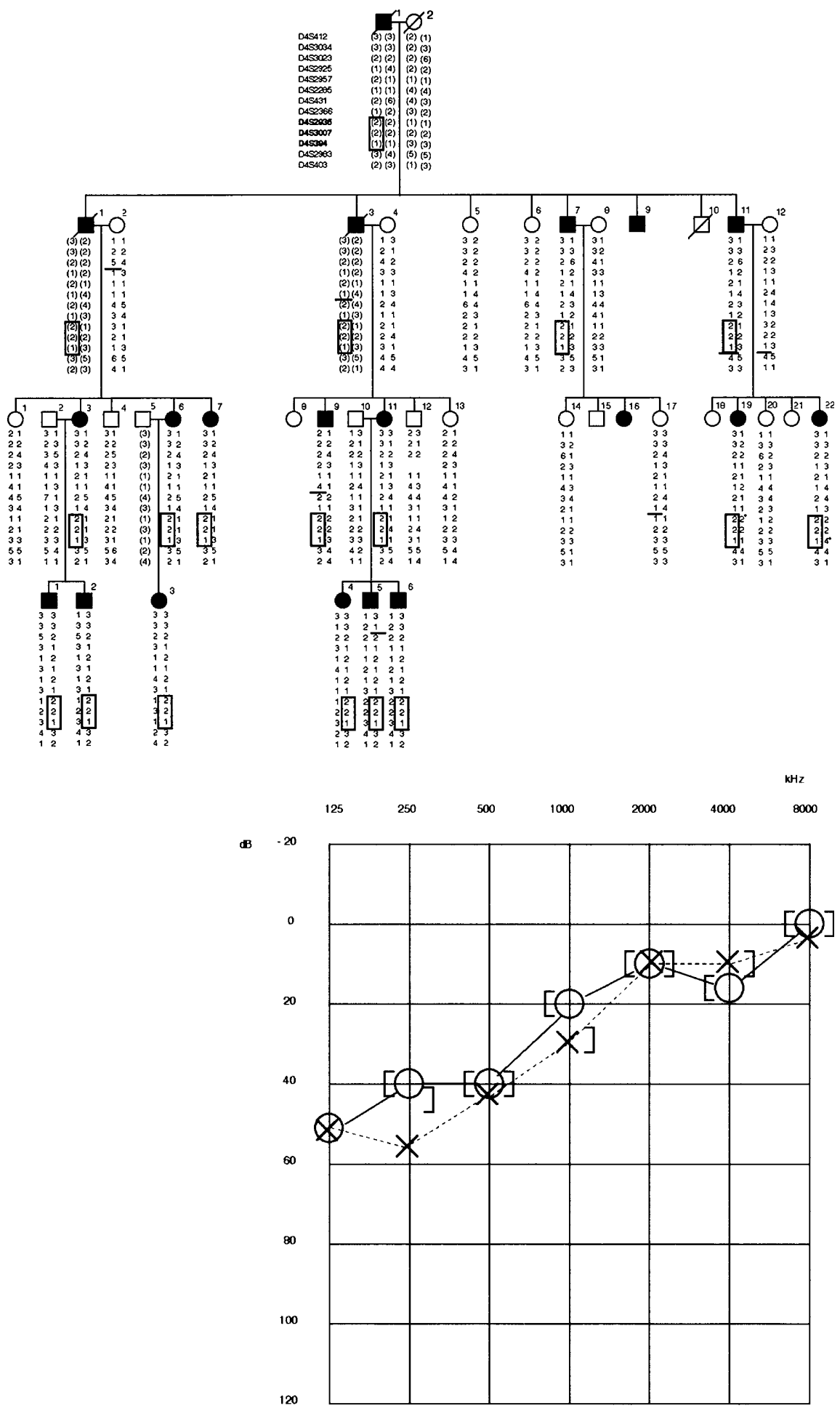

b

The two-point linkage analysis gave a maximum LOD score of 5.36 at a recombination fraction $(\theta)$ of $0.05(P=1.00)$ at the D4S2983 locus on chromosome 4p16 (Table 2). Haplotype analysis demonstrated that all 15 affected members examined had a common haplotype, "2-2-1," at three marker loci: D4S2935, D4S3007, and D4S394 (Fig. 1a). A critical recombination occurred between D4S394 and D4S2983 in affected individual II-11, who carried the disease-associated haplotype. A telomeric boundary of the disease locus was estimated to be between D4S2366 and D4S2935 by another recombination observed in individual III-17. These results, with reference to previously reported data for DFNA6/14 (Lesperance et al. 1995; Van Camp et al. 1999), suggested that the disease of this family was associated with a new LFSNHL locus between D4S2366 and D4S2983. 
Table 2. LOD scores calculated at various recombination fractions $(P=1.00)$

\begin{tabular}{llrllllll}
\hline \multicolumn{7}{l}{ Locus } & \multicolumn{7}{l}{ Recombination fraction $(\theta)$} \\
\cline { 2 - 8 } & 0.00 & \multicolumn{1}{c}{0.01} & 0.05 & 0.10 & 0.15 & 0.20 & 0.30 & 0.40 \\
\hline D4S412 & $-\infty$ & -1.58 & 0.29 & 0.89 & 1.10 & 1.13 & 0.92 & 0.44 \\
D4S3034 & $-\infty$ & 0.34 & 1.47 & 1.73 & 1.73 & 1.61 & 1.16 & 0.55 \\
D4S2957 & $-\infty$ & 0.74 & 2.44 & 2.83 & 2.82 & 2.61 & 1.87 & 0.83 \\
D4S3023 & $-\infty$ & -0.05 & 0.53 & 0.69 & 0.71 & 0.67 & 0.50 & 0.26 \\
D4S2925 & $-\infty$ & 2.53 & 3.53 & 3.62 & 3.42 & 3.09 & 2.17 & 0.99 \\
D4S2285 & $-\infty$ & 0.70 & 1.83 & 2.09 & 2.07 & 1.93 & 1.42 & 0.68 \\
D4S431 & $-\infty$ & 4.52 & 4.79 & 4.52 & 4.11 & 3.60 & 2.40 & 1.02 \\
D4S2366 & $-\infty$ & 4.05 & 4.35 & 4.14 & 3.77 & 3.31 & 2.22 & 0.94 \\
D4S2935 & $-\infty$ & 1.83 & 2.27 & 2.25 & 2.08 & 1.85 & 1.24 & 0.52 \\
D4S3007 & 1.74 & 1.71 & 1.61 & 1.48 & 1.34 & 1.19 & 0.86 & 0.46 \\
D4S394 & 2.64 & 2.58 & 2.37 & 2.10 & 1.82 & 1.52 & 0.91 & 0.31 \\
D4S2983 & $-\infty$ & 5.10 & 5.36 & 5.07 & 4.63 & 4.09 & 2.83 & 1.35 \\
D4S403 & $-\infty$ & 4.23 & 4.53 & 4.31 & 3.95 & 3.49 & 2.41 & 1.13 \\
\hline
\end{tabular}

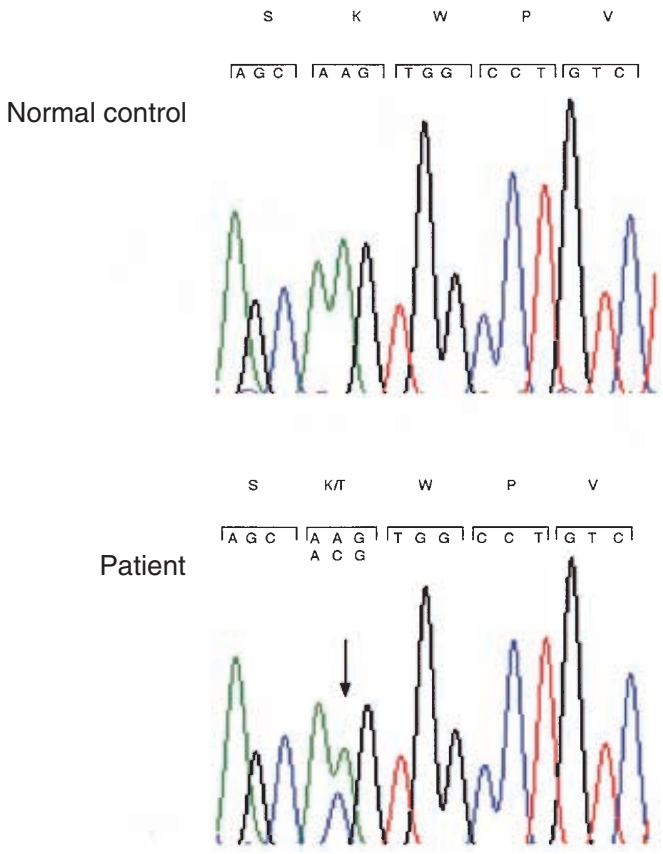

Fig. 2. Electropherogram showing a base substitution, AAG $\rightarrow$ ACG (arrow), at codon 634 in WFS1 exon 8, observed in affected person III-11

A WFS1 mutation in the family

No mutations were found in the ten genes other than WFS1 analyzed. However, in exon 8 of WFS1, a novel, heterozygous A-C transversion (AAG $\rightarrow$ ACG) was found in all affected members of the family. This missense mutation was predicted to lead to a lysine $\rightarrow$ threonine substitution at codon 634 (K634T) (Figs. 2, 3). None of the 196 normal controls had such an allele.

\section{Discussion}

In our initial linkage-haplotype analysis, LFSNHL was mapped to between $D 4 S 2366$ and $D 4 S 2983$ in this family. It

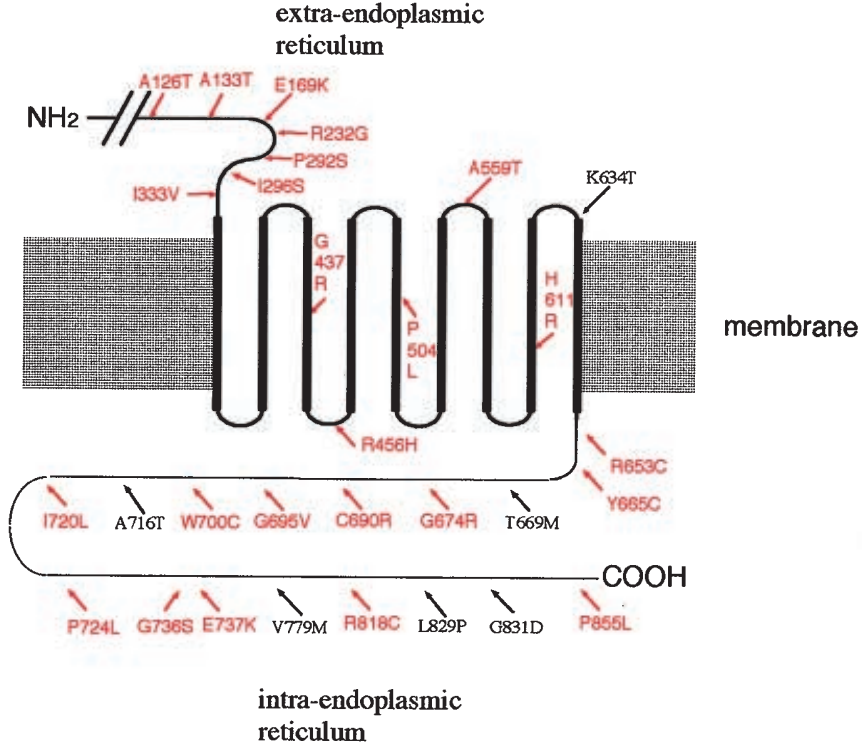

Fig. 3. Hypothetical structure of wolframin, and positions of mutations in LFSNHL families. The gray band indicates the membrane of the endoplasmic reticulum. The upper and lower sides show the extracytoplasmic and intracytoplasmic regions, respectively. Thin and thick lines denote the hydrophilic and transmembrame domains, respectively. Black arrows indicate the six known missense mutations found in seven families with DFNA6/14/38 (K634T, Japanese family; T699M, Dutch family; $A 716 T$, Dutch and American families; $V 779 M$, $G 831 D, L 829 P$, each in an American family). Red arrows indicate 23 known missense mutations associated with Wolfram syndrome. The protein truncation mutations, such as nonsense or frameshift mutations, that have been found in patients with Wolfram syndrome are not indicated in this figure

would seem that WFS1 is excluded from candidacy for the causative gene of the LFSNHL in this family because WFS1 is located between D4S2285 and D4S431. However, since no mutations were found in the ten other candidate genes located in the mapped disease region, we chose to search for mutations in WFS1 and found a heterozygous, missense mutation in the gene in all affected members of the family. Although the reasons why WFS1 was excluded from candidate region in our family remain unknown, possibilities 
include typing errors, spontaneous mutations in the microsatellite marker sequences used, and/or an inversion polymorphism. Among these, an inversion polymorphism around D4S431 and D4S2366 may be the most plausible explanation because many recombinations have been reported to have occurred around D4S431 (Bespalova et al. 2001; Inoue et al. 1998; Van Camp et al. 1999; Young et al. 2001). Such a polymorphism may have led to an inverted order of markers, which consequently resulted in an incorrect localization of the disease being mapped by the linkage analysis, away from the critical region.

WFS1, in which a missense mutation was observed in our patients, is the causative gene for Wolfram syndrome (WFS, OMIM "222300). WFS is an autosomal recessive disorder characterized by diabetes inspidus, juvenile-onset diabetes mellitus, bilateral progressive optic atrophy, and deafness. WFS1 encodes wolframin, which consists of 890 amino acids with nine putative transmembrane domains, a hydrophilic amino terminus, and a hydrophobic $\mathrm{COOH}$ tail, as determined by hydrophobicity analysis (Lesperance et al. 1995; Inoue et al. 1998; Strom et al. 1998). Many WFS1 mutations result in protein truncation, and some mutations result in amino acid substitutions. Five previously reported mutations (T699M, A716T, V799M, L829P, and G831D) in six DFNA6/14 patients were located in either the hydrophilic or the intracytoplasmic region in the transmembrane domains (Strom et al. 1998) (Fig. 3). The mutation site in the hydrophobic, extracytoplasmic, juxta-transmembrane region seen in this family was hitherto undescribed. This unique mutation site in our patients may be related to their milder phenotype compared with those of the six previously identified DFNA6/14 patients with WFS1 mutations (Lesperance et al. 1995; Strom et al. 1998). It is likely that a genotype-phenotype correlation is also applicable in the case of this disorder.

Wolframin has been reported to be localized to the endoplasmic reticulum in cultured cells, and its expression in the brain is restricted to certain populations of neurons, including the ventral cochlear nucleus and the inferior colliculus (Takeda et al. 2001). However, its expression in the peripheral auditory system remains unknown. Neuropathological studies of Wolfram syndrome have revealed loss of nerve fibers in the cochlear nerves as well as mild neuronal loss and gliosis in the auditory brainstem (Genis et al. 1997). These findings may indicate that wolframin plays an important role in the survival of neurons in specific areas, such as in a subpopulation of the afferent nerves from the cochlea. All six known WFS1 mutations in LFSNHL are heterozygous, missense mutations in the intra- or extracytoplasmic domain, and, they may thus cause a decrease in wolframin function, whereas in the case of Wolfram syndrome, its function may be abolished. Clarification of why such clinical differences are observed between the two disorders from a viewpoint of protein function requires further studies.
Acknowledgments We thank all family members for participating in this study. We also thank Ms. Kazumi Miyazaki, Naoko Takaki, Yasuko Noguchi, and Naoko Yanai for their excellent technical assistance. This study was supported by CREST, Japan Science and Technology Corporation (JST) and in part by Grants-in-Aid for Scientific Research Nos. 13203002, 13671787, 1367014, 12204140, and 13854024 from the Ministry of Education, Culture, Sports, Science and Technology of Japan.

\section{References}

Bespalova IN, Van Camp G, Bom SJ, Brown DJ, Cryns K, DeWan AT, Erson AE, Flothmann K, Kunst HP, Kurnool P, Sivakumaran TA, Cremers CW, Leal SM, Burmeister M, Lesperance MM (2001) Mutations in the Wolfram syndrome 1 gene (WFS1) are a common cause of low-frequency sensorineural hearing loss. Hum Mol Genet 10:2501-2508

Cohen MM, Gorlin RJ (1995) Epidemiology, etiology, and genetic patterns. In: Gorlin RJ, Toriello HV, Cohen MM (eds) Hereditary hearing loss and its syndromes. Oxford University Press, New York, pp 9-21

Cottingham RW Jr, Idurg RM, Schaffer AA (1993) Faster sequential genetic linkage computations. Am J Hum Genet 53:252263

Genis D, Davalos A, Molins A, Ferrer I (1997) Wolfram syndrome: a neuropathological study. Acta Neuropathol (Berl) 93:426-429

Inoue $\mathrm{H}$, Tanizawa $\mathrm{Y}$, Wasson J, Behn P, Kalidas K, Bernal-Mizrachi E, Mueckler M, Marshall H, Donis-Keller H, Crock P, Rogers D, Mikuni M, Kumashiro H, Higashi K, Sobue G, Oka Y, Permutt MA (1998) A gene encoding a transmembrane protein is mutated in patients with diabetes mellitus and optic atrophy (Wolfram syndrome). Nat Genet 20:143-148

Lesperance MM, Hall JW 3rd, Bess FH, Fukushima K, Jain PK, Ploplis B, San Agustin TB, Skarka H, Smith RJ, Wills M, Wilcox ER (1995) A gene for autosomal dominant nonsyndromic hereditary hearing impairment maps to 4p16.3. Hum Mol Genet 4:19671972

Lynch ED, Lee MK, Morrow JE, Welcsh PL, Leon PE, King MC (1997) Nonsyndromic deafness DFNA1 associated with mutation of a human homolog of the Drosophila gene diaphanous. Science 278:1315-1318

Sambrook J, Russel I (2001) Molecular cloning. Cold Spring Harbor Laboratory Press, New York

Schaffer AA, Gupta SK, Shriram K, Cottingham RW Jr (1994) Avoiding recomputation in linkage analysis. Hum Hered 44:225-237

Strom TM, Hortnagel K, Hofmann S, Gekeler F, Scharfe C, Rabl W, Gerbitz KD, Meitinger T (1998) Diabetes insipidus, diabetes mellitus, optic atrophy and deafness (DIDMOAD) caused by mutations in a novel gene (wolframin) coding for a predicted transmembrane protein. Hum Mol Genet 7:2021-2028

Takeda K, Inoue H, Tanizawa Y, Matsuzaki Y, Oba J, Watanabe Y, Shinoda K, Oka Y (2001) WFS1 (Wolfram syndrome 1) gene product: predominant subcellular localization to endoplasmic reticulum in cultured cells and neuronal expression in rat brain. Hum Mol Genet 10:477-484

Van Camp G, Kunst H, Flothmann K, McGuirt W, Wauters J, Marres H, Verstreken M, Bespalova IN, Burmeister M, Van de Heyning PH, Smith RJ, Willems PJ, Cremers CW, Lesperance MM (1999) A gene for autosomal dominant hearing impairment (DFNA14) maps to a region on chromosome $4 \mathrm{p} 16.3$ that does not overlap the DFNA6 locus. J Med Genet 36:532-536

Young TL, Ives E, Lynch E, Person R, Snook S, MacLaren L, Cator T, Griffin A, Fernandez B, Lee MK, King MC (2001) Non-syndromic progressive hearing loss DFNA38 is caused by heterozygous missense mutation in the Wolfram syndrome gene WFS1. Hum Mol Genet 10:2509-2514 